OPEN ACCESS

Edited by:

Julian Macoveanu,

Copenhagen University Hospital,

Denmark

Reviewed by:

Russell W. Chan,

Stanford University, United States

Ahmed Shalaby,

University of Louisville, United States

*Correspondence:

Bolun Wang

wangbolun@csu.edu.cn

Haishan Wu

wuhaishan@csu.edu.cn

${ }^{\dagger}$ These authors have contributed equally to this work

Specialty section:

This article was submitted to Mood and Anxiety Disorders,

a section of the journal

Frontiers in Psychiatry

Received: 05 May 2020

Accepted: 30 July 2020

Published: 14 August 2020

Citation:

Shan X, Qiu Y, Pan P, Teng Z, Li S, Tang $\mathrm{H}$, Xiang $\mathrm{H}, \mathrm{Wu} \mathrm{C}$, Tan $\mathrm{Y}$, Chen J, Guo W, Wang B and Wu H (2020)

Disrupted Regional

Homogeneity in Drug-Naive Patients With Bipolar Disorder.

Front. Psychiatry 11:825.

doi: 10.3389/fpsyt.2020.00825

\title{
Disrupted Regional Homogeneity in Drug-Naive Patients With Bipolar Disorder
}

\author{
Xiaoxiao Shan ${ }^{1 \dagger}$, Yan Qiu ${ }^{1 \dagger}$, Pan Pan ${ }^{1}$, Ziwei Teng ${ }^{1}$, Sujuan $\mathrm{Li}^{1}$, \\ Hui Tang ${ }^{1}$, Hui Xiang ${ }^{1}$, Chujun Wu ${ }^{1}$, Yuxi Tan ${ }^{1}$, Jindong Chen ${ }^{1}$, Wenbin Guo ${ }^{1,2}$, \\ Bolun Wang ${ }^{3 *}$ and Haishan Wu ${ }^{1 *}$ \\ ${ }^{1}$ National Clinical Research Center for Mental Disorders, and Department of Psychiatry, The Second Xiangya Hospital of \\ Central South University, Changsha, China, ${ }^{2}$ Department of Psychiatry, The Third People's Hospital of Foshan, Foshan, \\ China, ${ }^{3}$ Department of Orthopedics, The Second Xiangya Hospital, Central South University, Changsha, China
}

Objective: Studies on alterations in the regional neural activity in the brain of patients with bipolar disorder (BD) have provided conflicting results because of different medications used and study designs. A low bone mineral density (BMD) is also observed in patients with BD. This study aimed to further explore regional neural activities in unmedicated patients with $\mathrm{BD}$ and their association with $\mathrm{BMD}$.

Methods: In this study, 40 patients with BD and 42 healthy controls were scanned through resting-state functional magnetic resonance imaging (fMRI). Imaging data were analyzed with regional homogeneity (ReHo) and pattern classification. Pearson's correlation analyses were performed to explore the correlations between abnormal ReHo and BMD.

Results: A significant increase in ReHo values in the left inferior frontal gyrus (IFG)/ temporal pole, left cerebellum vermis I/vermis II/parahippocampal gyrus/brainstem, and right superior temporal gyrus (STG) and a decrease in ReHo in the occipital gyrus (OG; left middle OG/superior OG/bilateral cuneus) were found in the patients with $B D(p<0.05)$ compared with those in the healthy controls. No significant correlation was observed between the abnormal ReHo values in any of the brain regions of the patients with BMD.Support vector machine (SVM) analyses revealed that the ReHo values in the right STG for distinguishing patients from healthy controls showed an accuracy of $91.89 \%$, a sensitivity of $75.68 \%$, and a specificity of $83.78 \%$. The ReHo values in the left cerebellum vermis I/vermis II/parahippocampal gyrus/brainstem indicated an accuracy of $78.38 \%$, a sensitivity of $75.68 \%$, and a specificity of $81.08 \%$. 
Conclusion: This study further confirms the abnormal brain activities in extensive regions, and these brain regions are primarily located in the fronto-temporal-occipital circuit and the cerebellum vermis of patients with $\mathrm{BD}$. The regional neural activity in the right STG and the left cerebellum vermis I/vermis II/parahippocampal gyrus/brainstem may serve as potential imaging markers to distinguish patients with $\mathrm{BD}$ from healthy controls.

Keywords: bipolar disorder, regional homogeneity, support vector regression, magnetic resonance imaging, bone mineral density

\section{INTRODUCTION}

Bipolar disorder (BD) is a chronic, debilitating mood disorder associated with a high suicide risk $(1,2)$. BD is characterized by its mood swings between periods of depression and manic (BD type I) or hypomanic (BD type II) episodes (3). Patients with BD can be misdiagnosed as having unipolar depression if they only experience depressive episodes with no history of mania. Such patients are at a risk of inadequate treatment and poor clinical outcomes $(4,5)$.

Many neuroimaging studies have been performed to improve the accuracy of the diagnosis of BD. Despite the mixed results, some brain regions have been identified to present abnormal activities and functional connectivity (FC) (6-10). Three studies have revealed the increased functional connections of the amygdala, medial prefrontal cortex, and ventrolateral prefrontal cortex and a decreased connectivity between the medial prefrontal and cingulate cortices (6-8). The enhanced connectivity of the ventral striatum seed area with the orbitofrontal cortex and amygdala has also been reported in reward processing reception in remitted $\mathrm{BD}$ type $\mathrm{I}(9,10)$. The analytical techniques used in these neuroimaging studies are FC analysis and independent component analysis (ICA) that are efficient in exploring the function of brain networks but not local brain activities.

Regional homogeneity ( $\mathrm{ReHo}$ ) is a data-driven method to determine the degree of homogeneity within clusters through resting-state functional magnetic resonance imaging (fMRI) (11, 12). One advantage of ReHo is the ability to capture regional brain activities, compensating for the disadvantage of FC and ICA. Kendall's coefficient concordance (KCC) is commonly used in ReHo to measure the degree of the synchronization or similarity of a given voxel time series to that of its nearest neighbours through a voxel-wise method (12). An abnormal ReHo may reflect the temporal disruption of neural activities (13) and is associated with the pathophysiology of mental disorders (14). ReHo analysis has a high test-retest reliability (15) and has been applied to explore the regional abnormalities of functional synchronization in a range of psychiatric disorders, such as schizophrenia $(16,17)$, attention deficit hyperactivity disorder (18), and depression (19).

Previous studies with ReHo in BD (20-22) revealed abnormal neural activities in extensive brain regions, including the limbic system and temporal, frontal, and parietal lobes. However, few consistent abnormalities have been reported in relation to brain regions, such as the superior temporal gyrus (STG) and the middle frontal gyrus (MFG) $(20,21)$. Yao et al. found an increased ReHo in the left orbital inferior frontal gyrus (OIFG) and MFG and a decreased ReHo in the IFG, insula, STG, and occipital cortex of patients with BD I and II (20). Other studies have shown that pediatric patients with BD present a decreased ReHo in the middle temporal gyrus, bilateral MFG, and medial frontal gyrus (21) and that manic and euthymic pediatric patients with $\mathrm{BD}$ have differences in ReHo signals in the insula, STG, and cerebellum crus I (23). In addition, a decreased ReHo in the left OFG and an increased ReHo in the right supplementary motor area, bilateral middle occipital gyrus (OG), and right precentral gyrus are observed in unmedicated patients with BD II (24). The severity of clinical symptoms in patients with bipolar depression may be correlated with the consistency in the activation levels of the right cerebellum fusiform gyrus, left insula, and cerebellar vermis-related areas (25). Several factors, including concomitant drug administration, different $\mathrm{BD}$ states, types of $\mathrm{BD}$, illness duration, age range, and small sample size, may have contributed to the inconsistency of these findings. Furthermore, whether ReHo is associated with clinical symptoms remains unknown.

Psychosis may be predicted on the basis of neuroanatomical biomarkers by applying multivariate pattern recognition techniques, such as support vector machine (SVM), allowing predictions at an individual level. SVM has also been applied to differentiate patients with schizophrenia (26), prodromal syndrome (27), and BD (28) from healthy controls.

Previous studies revealed that $\mathrm{BD}$ is associated with a relatively low bone mineral density (BMD), leading to an increased risk of fractures in patients with BD (29-31). A recent systematic review has shown an increased fracture risk $(20 \%-80 \%)$ in patients with $\mathrm{BD}$ regardless of age, sex, comorbidities, and medication use (30). Nonetheless, the underlying mechanism related to the low BMD levels in $\mathrm{BD}$ remains unclear. Though chronic inflammation may play a role in bone loss through inflammation regulation in the central nervous system $(32,33)$, whether $\mathrm{BMD}$ is associated with the local neural activities of the brain of patients with $\mathrm{BD}$ has yet to be reported.

In the present study, we aimed to use ReHo to analyze the brain activity abnormalities in unmedicated patients with BD. We hypothesized that patients with BD would exhibit significantly abnormal ReHo values in specific brain regions, particularly in the prefrontal and temporal regions. We also proposed that ReHo could be employed as a potential imaging biomarker to differentiate patients with $\mathrm{BD}$ from healthy subjects. 


\section{MATERIALS AND METHODS}

\section{Participants}

All the patients with BD II aged 16-45 years were recruited from the Second Xiangya Hospital of Central South University, China, from March to November 2019. BD II was diagnosed by two experienced psychiatrists with the Structural Clinical Interview for Diagnostic and Statistical Manual of Mental Disorders, Fifth Edition (DSM-5) and Mini-Structured Clinical Interview for DSM-5 (SCID) and met the diagnostic criteria of BD II of DSM-5. The duration of BD II from onset did not exceed 5 years. All the patients were first diagnosed with BD II and had never taken any psychiatric medications. The inclusion criteria of BD II were as follows: 1) at least one hypomanie episode in the disease course (Criteria of "Hypomanic Episode" in DSM-5) and at least one major depressive episode in the disease course (Criteria of "Major Depressive Episode" in DSM-5); and 2) no history of a manic episode. Clinical symptoms were assessed using the Hamilton Depression Rating Scale-17 (HAMD-17), the Young Mania Rating Scale (YMRS), and the Hamilton Anxiety Rating Scale-17 (HAMA-17). The cognitive function was evaluated through the repeatable battery neuropsychologieal status (RBANS), including 12 subtests of cognitive functions; as such, the indices of delayed memory, immediate memory, language, visuospatial/constructional factors, and attention were obtained. The exclusion criteria were as follows: 1) other psychiatric disorders in accordance with the DSM-5; 2) any severe physical diseases, such as cardiovascular, kidney, or liver diseases; 3) any neuropsychiatric disorders; 4) any form of a traumatic brain injury; 5) history of electroconvulsive therapy; 6) drug or alcohol addiction; 7) pregnancy; and 8) contraindications for MRI scan.

Age-matched healthy controls were recruited from the local community through advertisements. The healthy subjects were screened using the Structured Clinical Interview for DSM-5-Nonpatient Version. The exclusion criteria were as follows: 1) any psychosis symptoms, neurological disease, or substance abuse, and 2) first-degree relatives having a history of psychiatric illness.

The study was approved by the ethics committee of the Second Xiangya Hospital of Central South University and performed in accordance with the Helsinki Declaration. All the participants provided written informed consent after a complete explanation. Additional written informed consent was obtained from the parents of patients aged below 18 years.

\section{BMD Measurement}

The BMDs in the left hip and the lumbar spine (L1-L4), including Ward's triangle (Ward's), femoral neck (Neck), and trochanter (Troch) of the patients with $\mathrm{BD}$ were measured through dual-energy X-ray absorptiometry (Discovery Wi; S/N 87556, US).

A quantifiable indicator of osteoporosis and low bone mass (34) was used and categorized as normal (T-score of -1 and above), low bone mass ( $\mathrm{T}$-score between -1 and -2.5), or osteoporosis (T-score below -2.5). The entire BMD measurement was carried out in the Second Xiangya Hospital of Central South University.

\section{Image Acquisition and Processing}

A 3.0 T Siemens scanner (Germany) was used for MRI scanning. The participants were asked to remain relaxed, supine, still, with eyes closed, and awake during the whole scanning. Foam pads and soft earplugs were used to reduce noise and scanner head motion. The scanner parameters were as follows: repetition time/ echo time $=2000 / 30 \mathrm{~ms}, 33$ axial slices, $64 \times 64$ matrix, $90^{\circ}$ flip angle, $22 \mathrm{~cm}$ field of view, $4 \mathrm{~mm}$ section thickness, $0.6 \mathrm{~mm}$ slice gap, and 240 volumes.

Data were preprocessed by the Data Processing Assistant for Resting-State fMRI software (DPARSF). The first 10 images were excluded from the analysis because of the instability of the initial MRI signal and for the participants to adapt to circumstances. The fMRI images were corrected for the acquisition delay between slices and head motion. All the subjects had no more than $2 \mathrm{~mm}$ of the translation in the $\mathrm{x}^{-}, \mathrm{y}^{-}$, or $\mathrm{z}$-axes and $2^{\circ}$ of rotation in each axis. The imaging data were then spatially normalized to the standard MNI EPI template in SPM8 and resampled to $3 \mathrm{~mm} \times 3 \mathrm{~mm} \times 3 \mathrm{~mm}$. Finally, the imaging data were linearly detrended and temporally band-pass-filtered (0.01-0.08 $\mathrm{Hz}$ ) to decrease the effect of low-frequency drifts and physiological high-frequency noise.

\section{ReHo Analysis}

ReHo was analyzed using REST (http://resting-fmri.sourceforge. net). The ReHo maps of each participant were acquired by calculating the KCC of a given voxel time series with those of its nearest neighbours. The KCC among the voxels was divided to normalize the ReHo maps through the mean KCC of the entire brain. The Gaussian kernel of $4 \mathrm{~mm}$ full-width at half maximum was used to spatially smoothen the generated ReHo maps.

\section{Statistical Analysis}

The demographics and clinical characteristics of the two groups were analyzed using two-sample-t-tests, independent t-tests, or a chi-square test when necessary. Differences in ReHo between patients and healthy controls were compared using voxel-wise two-sample t-tests with age and years of education as covariates. Gaussian random field theory was applied to correct multiple comparisons by using REST at $\mathrm{p}<0.05$ (voxel significance, $\mathrm{p}<$ 0.001; cluster significance, $\mathrm{p}<0.05$ ).

\section{Correlation Analysis}

When significant differences in ReHo were identified in the brain regions between the two groups, the average ReHo values were extracted from these specific regions. The correlations between abnormal ReHo values and clinical parameters were determined through Pearson's correlation analyses with the threshold for significance of $\mathrm{p}<0.05$. Bonferroni correction was employed to limit the type I error associated with multiple comparisons.

\section{Classification Analysis}

SVM was applied to test the capacity of the extracted ReHo in any brain region to discriminate patients with $\mathrm{BD}$ from the controls by using the LIBSVM software package (http://www. 
TABLE 1 | Characteristics of the subjects.

\begin{tabular}{|c|c|c|c|c|}
\hline & $\begin{array}{l}\text { Patients } \\
(n=37)\end{array}$ & $\begin{array}{l}\text { Controls } \\
(n=37)\end{array}$ & $\chi^{2} / \mathrm{T}$ & $\begin{array}{c}\mathrm{p}- \\
\text { value }\end{array}$ \\
\hline Sex (male/female) & $12 / 25$ & $17 / 20$ & 1.418 & $0.234^{a}$ \\
\hline Age (years) & $20.97 \pm 3.07$ & $20.84 \pm 3.11$ & -0.188 & $0.851^{b}$ \\
\hline Years of education (years) & $13.97 \pm 1.99$ & $14.65 \pm 2.04$ & 1.440 & $0.154^{b}$ \\
\hline \multicolumn{5}{|l|}{ BMD } \\
\hline $\mathrm{L} 1^{\mathrm{c}}$ & $0.90 \pm 0.12$ & & & \\
\hline $\mathrm{L}^{\mathrm{C}}$ & $1.32 \pm 2.04$ & & & \\
\hline $\mathrm{L}^{\mathrm{C}}$ & $1.00 \pm 0.11$ & & & \\
\hline$L 4^{\mathrm{C}}$ & $0.98 \pm 0.10$ & & & \\
\hline Total-lumbar ${ }^{\mathrm{c}}$ & $0.97 \pm 0.10$ & & & \\
\hline Neck $^{d}$ & $0.80 \pm 0.09$ & & & \\
\hline Troch $^{d}$ & $0.66 \pm 0.08$ & & & \\
\hline Ward's $^{d}$ & $0.77 \pm 0.12$ & & & \\
\hline HAMD- $17^{\mathrm{e}}$ & $22.19 \pm 6.86$ & & & \\
\hline HAMA- $17^{\mathrm{e}}$ & $25.36 \pm 8.16$ & & & \\
\hline YMRS & $8.08 \pm 5.51$ & & & \\
\hline Vocabulary learning ${ }^{f}$ & $28.53 \pm 4.75$ & & & \\
\hline Story retelling $^{f}$ & $13.85 \pm 4.59$ & & & \\
\hline $\begin{array}{l}\text { Immediate memory total } \\
\text { score }^{e}\end{array}$ & $40.03 \pm 12.67$ & & & \\
\hline Graphic copy ${ }^{\dagger}$ & $17.47 \pm 1.97$ & & & \\
\hline Line positioning $^{f}$ & $15.88 \pm 3.14$ & & & \\
\hline Visual span total score ${ }^{e}$ & $31.50 \pm 8.86$ & & & \\
\hline Picture named ${ }^{f}$ & $8.79 \pm 0.91$ & & & \\
\hline Verbal fluency test ${ }^{f}$ & $19.74 \pm 4.52$ & & & \\
\hline Verbal function total score ${ }^{e}$ & $26.94 \pm 8.10$ & & & \\
\hline Digit $\operatorname{span}^{f}$ & $14.79 \pm 1.77$ & & & \\
\hline Coding test ${ }^{f}$ & $57.24 \pm 10.48$ & & & \\
\hline Attention total score ${ }^{e}$ & $68.03 \pm 19.97$ & & & \\
\hline Vocabulary memory ${ }^{f}$ & $7.44 \pm 1.60$ & & & \\
\hline Vocabulary recognition $^{f}$ & $19.82 \pm 0.58$ & & & \\
\hline Story recall ${ }^{f}$ & $7.94 \pm 2.47$ & & & \\
\hline Figure memory ${ }^{\dagger}$ & $14.62 \pm 3.28$ & & & \\
\hline Delayed memory score ${ }^{e}$ & $47.06 \pm 12.88$ & & & \\
\hline Stroop word ${ }^{f}$ & $97.76 \pm 22.22$ & & & \\
\hline Stroop Color ${ }^{\dagger}$ & $69.35 \pm 18.76$ & & & \\
\hline Stroop Color-word ${ }^{f}$ & $41.91 \pm 9.13$ & & & \\
\hline
\end{tabular}

${ }^{a} p$ values were obtained by a chi-square test.

${ }^{b} p$ values were obtained by two-sample t-tests.

HAMD-17, Hamilton Depression Scale-17; HAMA-17, Hamilton anxiety Scale-17; YMRS, Young Mania Rating Scale; BMD, bone mineral density; Neck, femoral neck; Troch, trochanter of femoral; Ward's, Ward's triangle; ${ }^{c} n=33 ;{ }^{d} n=32 ;{ }^{e}=36 ;{ }^{f}=34$.

Among the 37 remaining patients, the HAMD scores of one patient were missing. The HAMA scores of another patient were missing. Five patients failed to complete the BMD measurement.

csie.ntu.edu.tw/ cjlin/libsvm/) in MATLAB (R2013b, The MathWorks, United States). The "leave-one-out" approach was implemented in the study.

\section{RESULTS}

\section{Clinical and Demographic Characteristics}

A total of 40 patients with BD II and 42 healthy controls were enrolled in this study. Of those, 37 patients with BD and 37 controls were included in the final analysis as the data of three patients and five controls were ruled out because of excessive head movements. No significant differences were observed in age, sex ratios, and years of education between the groups. The
TABLE 2 | Alterations in ReHo among patients with BD and controls.

\begin{tabular}{|c|c|c|c|c|c|}
\hline \multirow[t]{2}{*}{ Cluster location } & \multicolumn{3}{|c|}{ Peak (MNI) } & \multirow{2}{*}{$\begin{array}{l}\text { Number } \\
\text { of voxels }\end{array}$} & \multirow[t]{2}{*}{ T-value } \\
\hline & $\mathbf{x}$ & $\mathbf{y}$ & $\mathbf{z}$ & & \\
\hline $\begin{array}{l}\text { Left cerebellum vermis I/vermis II/ } \\
\text { parahippocampal gyrus/brainstem }\end{array}$ & 0 & -36 & -15 & 97 & 6.6861 \\
\hline Left IFG/temporal pole & -45 & 15 & -24 & 66 & 5.2961 \\
\hline Right STG & 48 & 12 & -21 & 61 & 5.6537 \\
\hline $\begin{array}{l}\text { Left middle OG/superior OG/bilateral } \\
\text { cuneus }\end{array}$ & -30 & -84 & 21 & 393 & -5.2520 \\
\hline
\end{tabular}

BD, bipolar disorder; ReHo, regional homogeneity; IFG, inferior frontal gyrus; OG, occipital gyrus; STG, superior temporal gyrus.

The significance level was set at $p<0.05$ for multiple comparisons corrected with Gaussian random field (GRF) theory (voxel significance, $p<0.001$; cluster significance, $p<0.05)$.

total BMD in the Total-lumbar, Ward's, Neck, and Troch were $0.97 \pm 0.10,0.77 \pm 0.12,0.80 \pm 0.09$, and $0.66 \pm 0.08$, respectively (Table 1).

\section{Differences in the ReHo Between Patients and Healthy Controls}

The ReHo in the left IFG/temporal pole, left cerebellum vermis I/ vermis II/parahippocampal gyrus/brainstem, and right STG of the patients with BD significantly increased compared with that of the controls. By contrast, a decreased ReHo was found in the OG (left middle OG/superior OG/bilateral cuneus; Table 2 and Figure 1).

\section{Correlation Results}

The ReHo values in the left middle OG/superior OG/bilateral cuneus were negatively correlated with the verbal function total score of RBANS $(r=-0.335, p=0.046)$, whereas the ReHo values in the left cerebellum vermis I/vermis II/parahippocampal gyrus/ brainstem were negatively correlated with the figure memory of RBANS ( $\mathrm{r}=-0.380, \mathrm{p}=0.026$ ) (Figure 2). However, these correlations were not significant after Bonferroni correction. No significant correlations were found between the ReHo values in any brain region and BMD levels of patients with BD (Supplemental Figures).

\section{SVM Analyses}

The ReHo values in the left cerebellum vermis I/vermis II/ parahippocampal gyrus/brainstem showed an accuracy of $78.38 \%$, a sensitivity of $75.68 \%$, and a specificity of $81.08 \%$ in SVM (Figure 3), whereas the ReHo values in the right STG showed an accuracy of $91.89 \%$, a sensitivity of $75.68 \%$, and a specificity of $83.78 \%$ (Figure 4 ). The detailed information of the SVM results are presented in Table 3.

\section{DISCUSSION}

This study found abnormal brain activities in patients with BD regarding ReHo and explored the relationship between the brain activity of specific regions and BMD levels. The results showed 


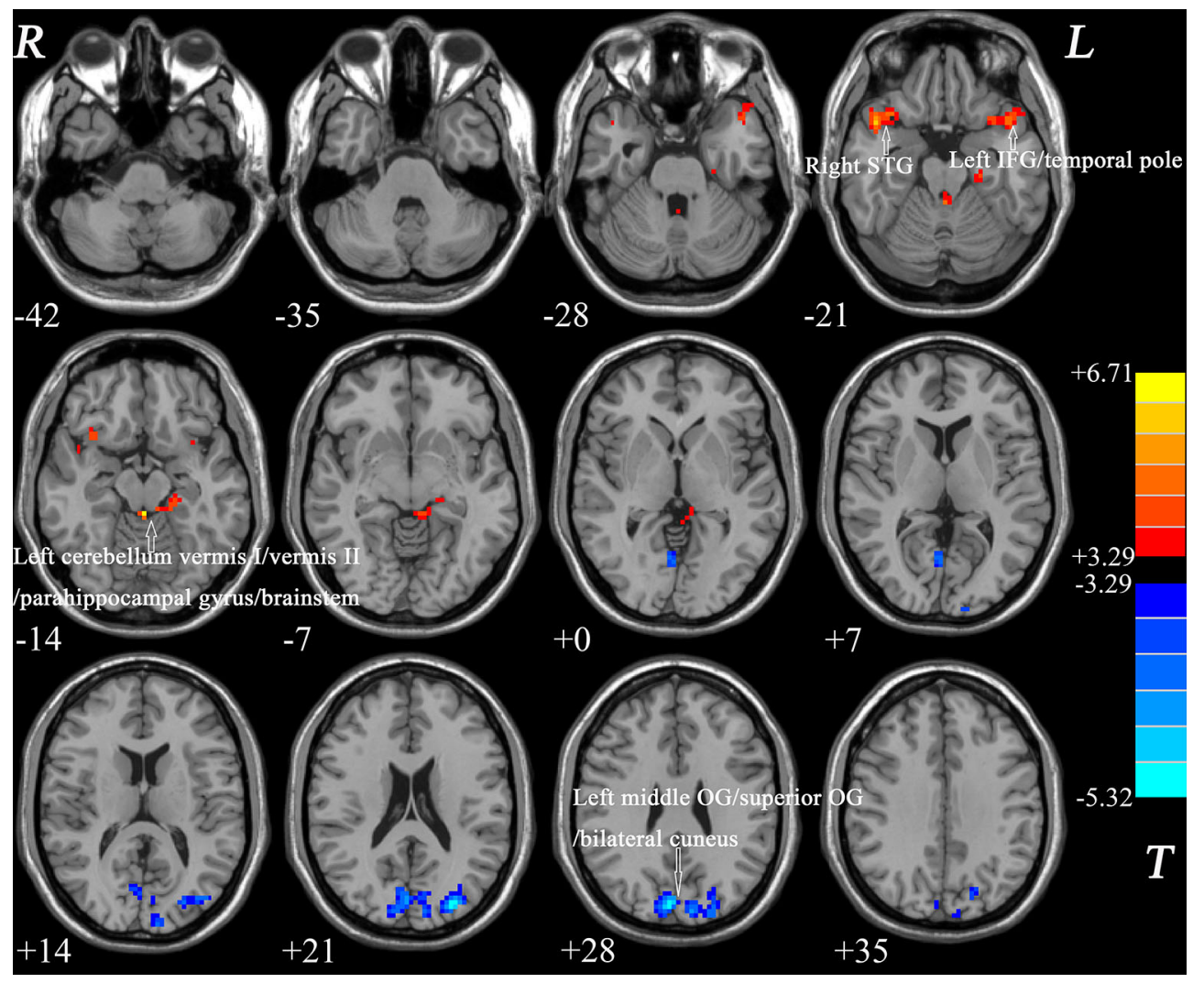

FIGURE 1 | Differences in ReHo between patients and healthy controls. Increased ReHo in the left IFG/temporal pole, left cerebellum vermis I/vermis II/ parahippocampal gyrus/brainstem, and right STG and a decreased ReHo in the OG (left middle OG/superior OG/bilateral cuneus) were observed in the patients with BD. The color bar represents the t values of the group analysis of ReHo. BD, bipolar disorder; ReHo, regional homogeneity; IFG, inferior frontal gyrus; STG, superior temporal gyrus; OG, occipital gyrus.

that the ReHo values in the left cerebellum vermis I/vermis II/ parahippocampal gyrus/brainstem, left IFG/temporal pole, and right STG increased, and the ReHo in the OG (left middle OG/ superior OG/bilateral cuneus) decreased. However, ReHo and $\mathrm{BMD}$ in any brain region were not significantly correlated. Furthermore, the SVM result showed that the ReHo values in the right STG and left cerebellum vermis I/vermis II/ parahippocampal gyrus/brainstem regions might be potential imaging markers to distinguish patients from healthy controls.

The frontal cortex has an established effect on emotional regulation, mental flexibility, impulsivity, self-awareness, and selfmonitoring (35). As part of the frontal cortex, the ventrolateral prefrontal cortex (including IFG) (7) and the orbitofrontal cortex (24) are also implicated in the pathophysiological features of BD. The IFG is considered to be crucially involved in the adjustment of emotional intensity and the integration of emotional information (36). Previous studies revealed the abnormal FC between the IFG and the medial prefrontal cortex, amygdala, and STG of patients with $\mathrm{BD}(8,37)$. The abnormal patterns of the FC between the IFG and nodes of the default network may also be associated with cognitive symptoms found in patients with $\mathrm{BD}$ (38). The functional dysconnectivity of the IFG with emotion-regulating regions, as a characteristic abnormality of $\mathrm{BD}$, can assist in clinical diagnosis (8). Our study further provided evidence on disturbed regional activities in IFG, suggesting the critical role of the ventrolateral prefrontal cortex in the neurological mechanism of $\mathrm{BD}$.

The temporal gyrus, as an auditory and visual-related brain region, is involved in the processing of facial emotions and working memory $(39,40)$. Structural and functional neuroimaging studies have revealed that $\mathrm{BD}$ is also associated with functional changes in the temporal gyrus (41). A decreased gray matter volume in the temporal gyrus has been reported in adult patients with BD (41). Several functional neuroimaging studies have indicated the dysfunction in related neural networks, which include the temporal gyrus, in emotional processing deficits $(42,43)$. A decreased connectivity between the amygdala and the temporal gyrus during an emotional face judgment task is found in pediatric patients with BD (44). Furthermore, the decreased ReHo values in the temporal gyrus have also been reported in patients with $\mathrm{BD}(20,21)$. The ReHo values obtained from the temporal gyrus were significantly different between patients with BD and unipolar depression; this result suggested that ReHo values could be a potential neuroimaging marker of $\mathrm{BD}(20)$. Our results implied that the 
A

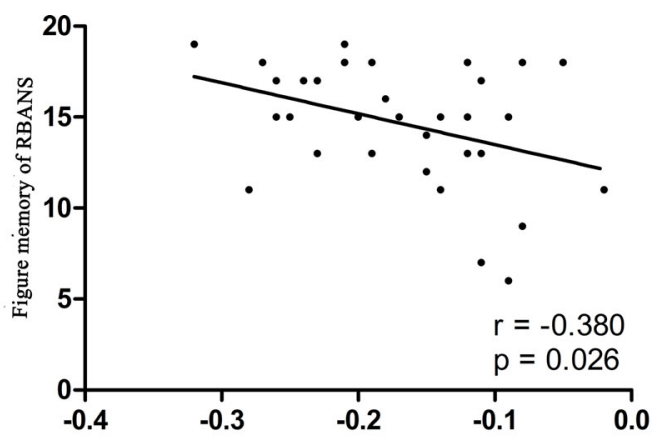

Left cerebellum vermis I/vermis II/parahippocampal gyrus/brainstem

B

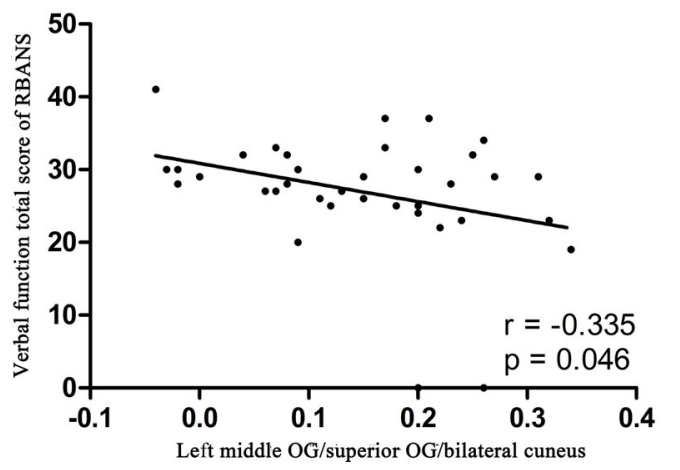

FIGURE 2 | Correlations between abnormal ReHo and cognition parameter scores. (A) Negative correlation was observed between the ReHo values in the left cerebellum vermis I/vermis II/parahippocampal gyrus/brainstem and the figure memory of patients with BD. (B) Negative correlation was observed between the ReHo values in the left middle OG/superior OG/bilateral cuneus and the verbal function total score of RBANS of patients with BD. BD, bipolar disorder; ReHo, regional homogeneity; OG, occipital gyrus; RBANS, repeatable battery neuropsychological status.

right temporal gyrus was presumably a part of the correlative functional network associated with BD.

In this study, the ReHo values in the occipital lobe decreased, suggesting a decrease in the local synchronization of spontaneous neural activities in the OG. OG has been revealed to be responsible for emotional facial adjustment as the main visual processing center $(45,46)$, particularly the middle OG that promotes interpersonal communication in specific social environments (47). In pediatric patients with $\mathrm{BD}$, the amplitude of the low-frequency fluctuation decreases in the bilateral inferior OG and left precuneus (48). The ReHo values in the OG, insula, and temporal cortex of patients with BD I or BD II also decrease, and this finding is consistent with our results (20). Structural MRI studies have revealed a decrease in the volume and thickness of the occipital cortex in patients with $\mathrm{BD}$ $(11,48)$. Moreover, they showed that the volume of the gray matter in the cuneus is associated with the performance of an inhibitory control task in patients with BD (49). Task-related fMRI results have also demonstrated the abnormal activation of
OG in patients with $\mathrm{BD}$ when they are asked to perform tasks involving emotional images (50), emotional face encoding (51), and sensory processing (52). Hence, the decreased ReHo values in OG found in this study might be associated with the emotional deficits commonly found in patients with BD.

The ReHo in the cerebellum vermis cortex, including vermis I/ vermis II, increased. Previous studies also reported abnormalities in the cerebellum cortex of patients with $\operatorname{BD}(23,25)$, suggesting that the cerebellum might also play a significant role in the pathophysiological features of $\mathrm{BD}$ (53). The cerebellum is interconnected with other prefrontal and limbic brain regions and may work cooperatively to regulate cognitive and emotional processing (54). Xiao et al (23) found an increase in ReHo in the right cerebellum crus1 in pediatric patients with $\mathrm{BD}$, and this observation also agrees with our finding about abnormalities in the cerebellum vermis.

A previous study revealed that a specificity or sensitivity greater than 0.7 is conducive to the establishment of diagnostic indicators (55). In contrast, a specificity or sensitivity of less than 0.6 may indicate a poor establishment of diagnostic indicators (56). SVM has been widely used in biomedical diagnoses (57). A valid radiomic method through resting-state fMRI can identify patients with $\mathrm{BD}$ from healthy controls with more than $80 \%$ of classification accuracy via SVM analysis (28). Therefore, this method is a potential adjunctive tool for clinical diagnostic systems. The SVM analysis in our study showed that the accuracy, sensitivity, and specificity for distinguishing patients from healthy controls were more than 0.7 in terms of the ReHo values of the right STG regions and the left cerebellum vermis I/ vermis II/parahippocampal gyrus/brainstem. Hence, the ReHo values in these brain regions could be employed as potential imaging markers to distinguish patients with $\mathrm{BD}$ from controls.

Our study also tried to explore the correlation between abnormal ReHo and BMD in BD. However, abnormal ReHo was not correlated with $\mathrm{BMD}$ in patients with $\mathrm{BD}$. Therefore, the potential mechanism underlying the low BMD of patients with BD remains unknown. Aspects such as inflammatory cytokines, endocrine factors, oxidative stress, and mitochondrial dysfunction have common pathways that can influence neuroprogression and lead to a low $\mathrm{BMD}$ in $\operatorname{BD}(58,59)$. Chronic neuroinflammation results in an increased level of free radicals, lipid peroxidation, reduced mitochondrial function, and excitatory toxicity, and all of them have neuroprogressive effects because of neurotoxicity (60). No significant correlation was found between ReHo and BMD possibly because of a relatively small sample size, and/or a low signal-to-noise ratio in resting-state fMRI.

Apart from the small sample size, other limitations in the current study should be considered. First, the abnormalities in gray/white matters were not evaluated, but whether these abnormalities were a basis of changes in ReHo remains unclear. Second, the use of the Montreal Neurological Institute template might be a potential confounding factor as the template was initially generated on the basis of a Caucasian population, so it might not fit the study on the Chinese population. Third, the resolution in our study was relatively lower than that of other resting-state fMRI studies, which might have limited the 
A

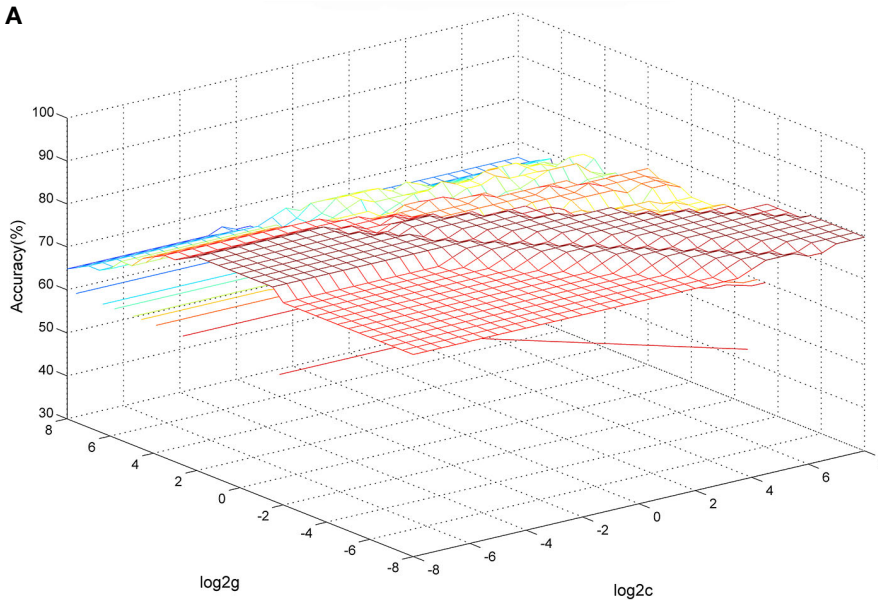

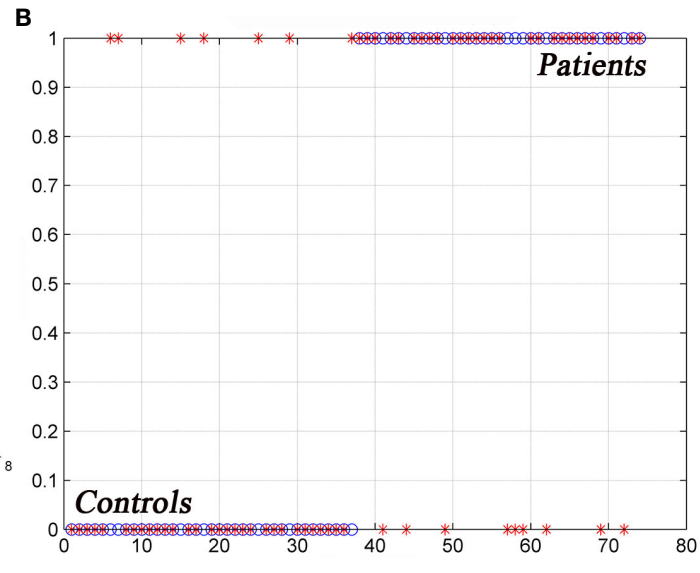

FIGURE 3 | Differentiating the patients from the controls by using the increased ReHo values in the left cerebellum vermis I/vermis II/parahippocampal gyrus/brainstem. Visualization of the classifications via a support vector machine (SVM) by using the ReHo values in significantly different regions. (A) result of the SVM parameter selection via a 3D view; (B) classified map of the ReHo values in the left cerebellum vermis I/vermis II/parahippocampal gyrus/brainstem. ReHo, regional homogeneity.
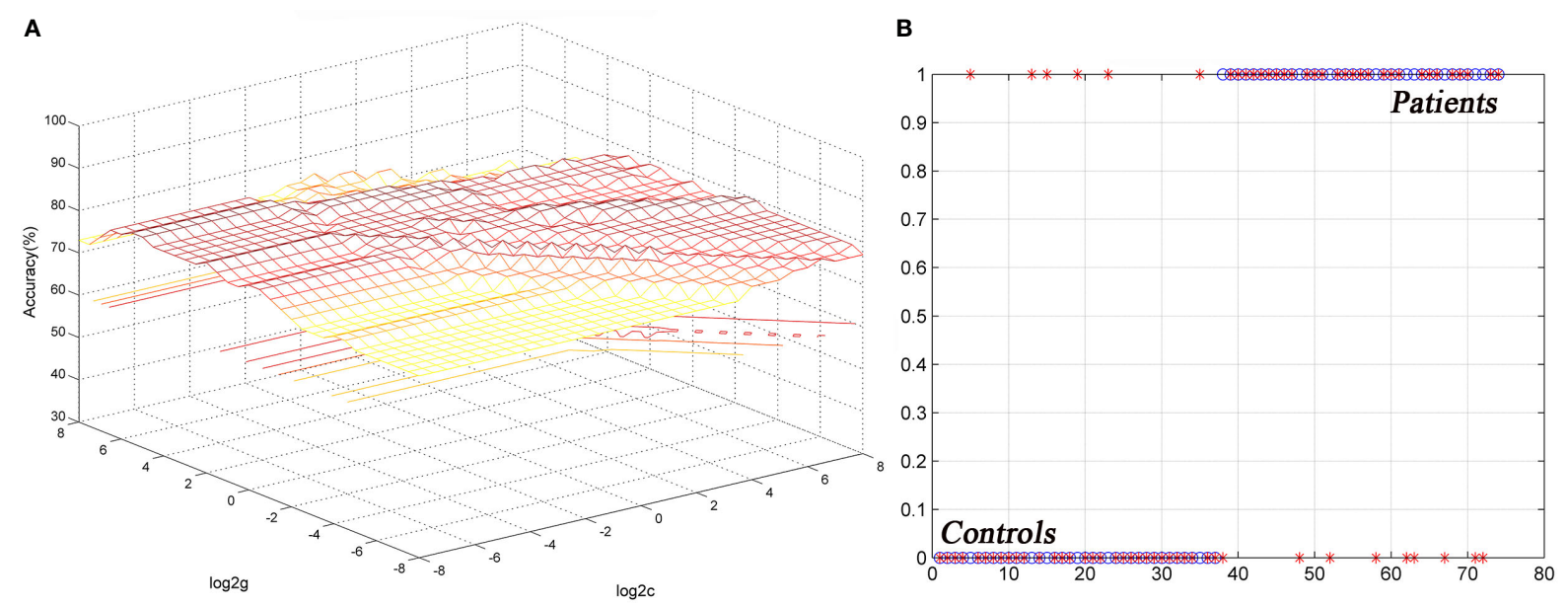

FIGURE 4 | Differentiating the patients from the controls by using the increased ReHo values in the right STG. Visualization of classifications via a support vector machine (SVM) by using the ReHo values in the significantly different regions. (A) result of SVM parameter selection via a 3D view; (B) classified map of the ReHo values in the right STG. ReHo, regional homogeneity; STG, superior temporal gyrus.

TABLE 3 | Differentiating the patients from the controls by using the ReHo values of a single region with the SVM method.

\begin{tabular}{|c|c|c|c|}
\hline Brain region & Sensitivity & Specificity & Accuracy \\
\hline Left cerebellum vermis I/vermis II/parahippocampal gyrus/brainstem & $75.68 \%(28 / 37)$ & $81.08 \%(30 / 37)$ & $78.38 \%(58 / 74)$ \\
\hline Right STG & $75.68 \%(28 / 37)$ & $83.78 \%(31 / 37)$ & $91.89 \%(68 / 74)$ \\
\hline Left IFG/temporal pole & $67.57 \%(25 / 37)$ & $72.97 \%(27 / 37)$ & $70.27 \%(52 / 74)$ \\
\hline Left middle OG/superior OG/bilateral cuneus & $62.16 \%(23 / 37)$ & $83.78 \%(31 / 37)$ & $72.97 \%(54 / 74)$ \\
\hline
\end{tabular}

ReHo, regional homogeneity; SVM, support vector machines; IFG, inferior frontal gyrus; OG, occipital gyrus; STG, superior temporal gyrus.

interpretation of the fMRI results. Lastly, ReHo was only limited to activity in local brain regions, failing to explore functional connections between whole brain regions. As a voxel-based method, its objectivity might cause potential registration or normalization errors. Hence, the present results should be interpreted with caution.

In conclusion, this study provided supporting evidence on disrupted regional neural activities in extensive regions and 
these brain regions primarily locate in the fronto-temporaloccipital circuit and the cerebellum vermis in patients with $\mathrm{BD}$ II during the resting state. The regional neural activity in the right STG and the left cerebellum vermis I/vermis II/ parahippocampal gyrus/brainstem might serve as potential imaging markers to distinguish patients with BD from healthy controls.

Future research should continue to examine patients with BD in large sample sizes through functional imaging and longitudinal studies to clarify the potential causal relationship between changes in ReHo and BD.

\section{DATA AVAILABILITY STATEMENT}

All datasets presented in this study are included in the article/ Supplementary Material.

\section{ETHICS STATEMENT}

The studies involving human participants were reviewed and approved by: The study was approved by the ethics committee of the Second Xiangya Hospital of Central South University. Our study was performed in accordance with the Helsinki Declaration. All the participants provided written informed consent. Additional written informed consent was obtained from the parents of patients aged below 18 years.

\section{REFERENCES}

1. Dong D, Ming Q, Zhong X, Pu W, Zhang X, Jiang Y, et al. State-independent alterations of intrinsic brain network in current and remitted depression. Prog Neuropsychopharmacol Biol Psychiatry (2019) 89:475-80. doi: 10.1016/ j.pnpbp.2018.08.031

2. Gong Q, Hu X, Pettersson-Yeo W, Xu X, Lui S, Crossley N, et al. Network-Level Dysconnectivity in Drug-Naive First-Episode Psychosis: Dissociating Transdiagnostic and Diagnosis-Specific Alterations. Neuropsychopharmacology (2017) 42:933-40. doi: 10.1038/npp.2016.247

3. Grande I, Berk M, Birmaher B, Vieta E. Bipolar disorder. Lancet (2016) 387:1561-72. doi: 10.1016/S0140-6736(15)00241-X

4. Houenou J, Frommberger J, Carde S, Glasbrenner M, Diener C, Leboyer M, et al. Neuroimaging-based markers of bipolar disorder: evidence from two meta-analyses. J Affect Disord (2011) 132:344-55. doi: 10.1016/j.jad. 2011.03.016

5. Bowden CL. Diagnosis, treatment, and recovery maintenance in bipolar depression. J Clin Psychiatry (2010) 71:e1. doi: 10.4088/JCP.8125cc5c

6. Perry A, Roberts G, Mitchell PB, Breakspear M. Correction: Connectomics of bipolar disorder: a critical review, and evidence for dynamic instabilities within interoceptive networks. Mol Psychiatry (2019) 24:1398. doi: 10.1038/ s41380-018-0327-7

7. Syan SK, Smith M, Frey BN, Remtulla R, Kapczinski F, Hall G, et al. Restingstate functional connectivity in individuals with bipolar disorder during clinical remission: a systematic review. J Psychiatry Neurosci (2018) 43:298316. doi: $10.1503 /$ jpn.170175

8. Roberts G, Lord A, Frankland A, Wright A, Lau P, Levy F, et al. Functional Dysconnection of the Inferior Frontal Gyrus in Young People With Bipolar Disorder or at Genetic High Risk. Biol Psychiatry (2017) 81:718-27. doi: 10.1016/j.biopsych.2016.08.018

\section{AUTHOR CONTRIBUTIONS}

All authors contributed to the article and approved the submitted version. XS and YQ contributed equally to this work, wrote the manuscript, and conducted the study. ZT, SL, HT, HX, CW, and YT collected the original imaging data. BW and HW designed the study. WG and JC managed and analyzed the imaging data.

\section{FUNDING}

This study was supported by grants from the National Natural Science Foundation of China (Grant Nos. 81971258 and 81270019).

\section{ACKNOWLEDGMENTS}

We would like to thank all the subjects who served as research participants.

\section{SUPPLEMENTARY MATERIAL}

The Supplementary Material for this article can be found online at: https://www.frontiersin.org/articles/10.3389/fpsyt.2020.00825/ full\#supplementary-material

9. Dutra SJ, Man V, Kober H, Cunningham W, Gruber J. Disrupted corticolimbic connectivity during reward processing in remitted bipolar I disorder. Bipolar Disord (2017) 19(8):661-75. doi: 10.1111/bdi.12560

10. Niu M, Wang Y, Jia Y, Wang J, Zhong S, Lin J, et al. Common and Specific Abnormalities in Cortical Thickness in Patients with Major Depressive and Bipolar Disorders. EBioMedicine (2017) 16:162-71. doi: 10.1016/j.ebiom. 2017.01.010

11. Zuo XN, Xu T, Jiang L, Yang $\mathrm{Z}$, Cao $\mathrm{X}$, He $\mathrm{Y}$, et al. Toward reliable characterization of functional homogeneity in the human brain: preprocessing, scan duration, imaging resolution and computational space. Neuroimage (2013) 65:374-86. doi: 10.1016/j.neuroimage.2012.10.017

12. Zang Y, Jiang T, Lu Y, He Y, Tian L. Regional homogeneity approach to fMRI data analysis. Neuroimage (2004) 22:394-400. doi: 10.1016/ j.neuroimage.2003.12.030

13. He Y, Wang L, Zang Y, Tian L, Zhang X, Li K, et al. Regional coherence changes in the early stages of Alzheimer's disease: A combined structural and resting-state functional MRI study. Neuroimage (2007) 35:488-500. doi: 10.1016/j.neuroimage.2006.11.042

14. Liu Z, Xu C, Xu Y, Wang Y, Zhao B, Lv Y, et al. Decreased regional homogeneity in insula and cerebellum: A resting-state fMRI study in patients with major depression and subjects at high risk for major depression. Psychiatry Res (2010) 182:211-5. doi: 10.1016/j.pscychresns. 2010.03.004

15. Zuo XN, Xing XX. Test-retest reliabilities of resting-state FMRI measurements in human brain functional connectomics: a systems neuroscience perspective. Neurosci Biobehav Rev (2014) 45:100-18. doi: 10.1016/j.neubiorev.2014.05.009

16. Yu R, Hsieh MH, Wang HS, Liu CM, Liu CC, Hwang T, et al. Frequency Dependent Alterations in Regional Homogeneity of Baseline Brain Activity in Schizophrenia. PLoS One (2013) 8(3):e57516. doi: 10.1371/journal. pone.0057516 
17. Wang S, Zhang Y, Lv L, Wu R, Fan X, Zhao J, et al. Abnormal regional homogeneity as a potential imaging biomarker for adolescent-onset schizophrenia: A resting-state fMRI study and support vector machine analysis. Schizophr Res (2018) 192:179-84. doi: 10.1016/j.schres.2017.05.038

18. Cao QJ, Zang YF and Wang YF. Brain functions in attention deficit hyperactivity disorder combined and inattentive subtypes: A resting-state functional magnetic resonance imaging study. Beijing Da Xue Xue Bao Yi Xue Ban (2007) 39:261-5.

19. Guo WB, Liu F, Xue Z, Yu Y, Ma CQ, Tan CL, et al. Abnormal neural activities in first-episode, treatment-naive, short-illness-duration, and treatment-response patients with major depressive disorder: A resting-state fMRI study. J Affect Disord (2011) 135(1-3):326-31. doi: 10.1016/j.jad.2011.06.048

20. Yao X, Yin Z, Liu F, Wei S, Zhou Y, Jiang X, et al. Shared and distinct regional homogeneity changes in bipolar and unipolar depression. Neurosci Lett (2018) 673:28-32. doi: 10.1016/j.neulet.2018.02.033

21. Gao W, Jiao Q, Lu S, Zhong Y, Qi R, Lu D, et al. Alterations of regional homogeneity in pediatric bipolar depression: a resting-state fMRI study. BMC Psychiatry (2014) 14:222. doi: 10.1186/s12888-014-0222-y

22. Liang MJ, Zhou Q, Yang KR, Yang XL, Fang J, Chen WL, et al. Identify changes of brain regional homogeneity in bipolar disorder and unipolar depression using resting-state FMRI. PLoS One (2013) 8:e79999. doi: 10.1371/ journal.pone.0079999

23. Xiao Q, Cui D, Jiao Q, Zhong Y, Cao W, Lu G, et al. Altered regional homogeneity in pediatric bipolar disorder during manic and euthymic state: a resting-state fMRI study. Brain Imaging Behav (2019) 13(6):1789-98. doi: 10.1007/s11682-019-00117-4

24. Qiu S, Chen F, Chen G, Jia Y, Gong J, Luo X, et al. Abnormal resting-state regional homogeneity in unmedicated bipolar II disorder. J Affect Disord (2019) 256:604-10. doi: 10.1016/j.jad.2019.06.037

25. Xu Z, Lai J, Zhang H, Ng C, Zhang P, Xu D, et al. Regional homogeneity and functional connectivity analysis of resting-state magnetic resonance in patients with bipolar II disorder. Med (Baltimore) (2019) 98(47):e17962. doi: 10.1097/MD.0000000000017962

26. Wang Y, Zhong S, Chen G, Liu T, Zhao L, Sun Y, et al. Altered cerebellar functional connectivity in remitted bipolar disorder: A resting-state functional magnetic resonance imaging study. Aust N Z J Psychiatry (2018) 52:962-71. doi: 10.1177/0004867417745996

27. Shan XX, Ou YP, Pan P, Ding YD, Zhao J, Liu F, et al. Increased frontal gray matter volume in individuals with prodromal psychosis. CNS Neurosci Ther (2019) 25:987-94. doi: 10.1111/cns.13143

28. Wang Y, Sun K, Liu Z, Chen G, Jia Y, Zhong S, et al. Classification of Unmedicated Bipolar Disorder Using Whole-Brain Functional Activity and Connectivity: A Radiomics Analysis. Cereb Cortex (2019) 30(3):1117-28. doi: 10.1093/cercor/bhz152

29. Chandrasekaran V, Brennan-Olsen SL, Stuart AL, Pasco JA, Berk M, Hodge JM, et al. Association between bipolar spectrum disorder and bone health: a meta-analysis and systematic review protocol. BMJ Open (2017) 7:e13981. doi: 10.1136/bmjopen-2016-013981

30. Chandrasekaran V, Brennan-Olsen SL, Stuart AL, Pasco JA, Berk M, Hodge JM, et al. Bipolar disorder and bone health: A systematic review. J Affect Disord (2019) 249:262-9. doi: 10.1016/j.jad.2019.02.013

31. Hsu CC, Hsu YC, Chang KH, Lee CY, Chong LW, Wang YC, et al. Increased risk of fracture in patients with bipolar disorder: a nationwide cohort study. Soc Psychiatry Psychiatr Epidemiol (2016) 51:1331-8. doi: 10.1007/s00127-016-1242-3

32. Redlich K, Smolen JS. Inflammatory bone loss: pathogenesis and therapeutic intervention. Nat Rev Drug Discov (2012) 11:234-50. doi: 10.1038/nrd3669

33. Stich O, Andres TA, Gross CM, Gerber SI, Rauer S. Langosch JM An observational study of inflammation in the central nervous system in patients with bipolar disorder. Bipolar Disord (2015) 17(3):291-302. doi: 10.1111/bdi. 12244

34. Kanis JAthe WHO Study Group. Assessment of fracture risk and its application to screening for postmenopausal osteoporosis. Report of a WHO Study Group. World Health Organ Tech Rep Ser (1994) 4(6):368-81. doi: $10.1007 / \mathrm{BF} 01622200$

35. Morgan KD, Dazzan P, Morgan C, Lappin J, Hutchinson G, Suckling J, et al. Insight, grey matter and cognitive function in first-onset psychosis. $\mathrm{Br} \mathrm{J}$ Psychiatry (2010) 197:141-8. doi: 10.1192/bjp.bp.109.070888

36. Foland-Ross LC, Bookheimer SY, Lieberman MD, Sugar CA, Townsend JD, Fischer J, et al. Normal amygdala activation but deficient ventrolateral prefrontal activation in adults with bipolar disorder during euthymia. Neuroimage (2012) 59(1):738-44. doi: 10.1016/j.neuroimage.2011.07.054

37. Torrisi S, Moody TD, Vizueta N, Thomason ME, Monti MM, Townsend JD, et al. Differences in resting corticolimbic functional connectivity in bipolar I euthymia. Bipolar Disord (2013) 15:156-66. doi: 10.1111/bdi.12047

38. Ricciardiello L, Fornaro P. Beyond the cliff of creativity: a novel key to Bipolar Disorder and creativity. Med Hypotheses (2013) 80:534-43. doi: 10.1016/ j.mehy.2012.12.018

39. Antonius D, Prudent V and Rebani Y, D'Angelo D, Ardekani BA, Malaspina D, et al. White matter integrity and lack of insight in schizophrenia and schizoaffective disorder. Schizophr Res (2011) 128:76-82. doi: 10.1016/ j.schres.2011.02.020

40. Pavuluri MN, Passarotti AM, Fitzgerald JM, Wegbreit E, Sweeney JA Risperidone and divalproex differentially engage the fronto-striato-temporal circuitry in pediatric mania: a pharmacological functional magnetic resonance imaging study. J Am Acad Child Adolesc Psychiatry (2012) 51:157-70. doi: 10.1016/j.jaac.2011.10.019

41. Moorhead TW, McKirdy J, Sussmann JE, Hall J, Lawrie SM, Johnstone EC, et al. Progressive gray matter loss in patients with bipolar disorder. Biol Psychiatry (2007) 62:894-900. doi: 10.1016/j.biopsych.2007.03.005

42. Adleman NE, Kayser RR, Olsavsky AK, Bones BL, Muhrer EJ, Fromm SJ, et al. Abnormal fusiform activation during emotional-face encoding assessed with functional magnetic resonance imaging. Psychiatry Res (2013) 212:161-3. doi: 10.1016/j.pscychresns.2013.01.006

43. Kleinhans NM, Richards T, Sterling L, Stegbauer KC, Mahurin R, Johnson LC, et al. Abnormal functional connectivity in autism spectrum disorders during face processing. Brain (2008) 131:1000-12. doi: 10.1093/brain/awm334

44. Rich BA, Fromm SJ, Berghorst LH, Dickstein DP, Brotman MA, Pine DS, et al. Neural connectivity in children with bipolar disorder: impairment in the face emotion processing circuit. J Child Psychol Psychiatry (2008) 49:88-96. doi: 10.1111/j.1469-7610.2007.01819.x

45. Thomas LA, Brotman MA, Bones BL, Chen G, Rosen BH, Pine DS, et al. Neural circuitry of masked emotional face processing in youth with bipolar disorder, severe mood dysregulation, and healthy volunteers. Dev Cognit Neurosci (2014) 8:110-20. doi: 10.1016/j.dcn.2013.09.007

46. Tillová K, Jurák P, Chládek J, Halámek J, Rektor I. The posterior medial cortex is involved in visual but not in verbal memory encoding processing: an intracerebral recording study. J Neural Transm (2013) 120(3):391-7. doi: 10.1007/s00702-012-0890-Z

47. Zhang X, Tang Y, Zhu Y, Li Y, Tong S. study of functional brain homogeneity in female patients with major depressive disorder. In: 2016 38th Annual International Conference of the IEEE Engineering in Medicine and Biology Society (EMBC) (2016). p. 2562-5. doi: 10.1109/EMBC.2016.7591253

48. Lu D, Jiao Q, Zhong Y, Gao W, Xiao Q, Liu X, et al. Altered baseline brain activity in children with bipolar disorder during mania state: a resting-state study. Neuropsychiatr Dis Treat (2014) 10:317-23. doi: 10.2147/NDT. S54663

49. Abe C, Ekman CJ, Sellgren C, Petrovic P, Ingvar M, Landén M. Cortical thickness, volume and surface area in patients with bipolar disorder types I and II. J Psychiatry Neurosci (2016) 41:240-50. doi: 10.1503/jpn.150093

50. Cerullo MA, Eliassen JC, Smith CT, Fleck DE, Nelson EB, Strawn JR, et al. Bipolar I disorder and major depressive disorder show similar brain activation during depression. Bipolar Disord (2014) 16:703-12. doi: 10.1111/bdi.12225

51. Tseng WL, Bones BL and Kayser RR, Olsavsky AK, Fromm SJ, Pine DS, et al. An fMRI study of emotional face encoding in youth at risk for bipolar disorder. Eur Psychiatry (2015) 30:94-8. doi: 10.1016/j.eurpsy.2014.05.004

52. Shaffer JJ, Johnson CP, Fiedorowicz JG, Christensen GE, Wemmie JA, Magnotta VA, et al. Impaired sensory processing measured by functional MRI in Bipolar disorder manic and depressed mood states. Brain Imaging Behav (2018) 12:83747. doi: 10.1007/s11682-017-9741-8

53. Kim D, Byul Cho H, Dager SR, Yurgelun-Todd DA, Yoon S, Lee JH, et al. Posterior cerebellar vermal deficits in bipolar disorder. J Affect Disord (2013) 150:499-506. doi: 10.1016/j.jad.2013.04.050

54. Stoodley CJ, Schmahmann JD. Evidence for topographic organization in the cerebellum of motor control versus cognitive and affective processing. Cortex (2010) 46:844. doi: 10.1016/j.cortex.2009.11.008

55. Swets JA. Measuring the accuracy of diagnostic systems. Science (1988) 240:1285-93. doi: 10.1126/science.3287615 
56. Gong Q, Wu Q, Scarpazza C, Lui S, Jia Z, Marquand A, et al. Prognostic prediction of therapeutic response in depression using high-field MR imaging. Neuroimage (2011) 55:1497-503. doi: 10.1016/j.neuroimage.2010.11.079

57. Wang H, Guo W, Liu F, Wang G, Lyu H, Wu R, et al. Patients with firstepisode, drug-naive schizophrenia and subjects at ultra-high risk of psychosis shared increased cerebellar-default mode network connectivity at rest. Sci Rep (2016) 6:26124. doi: 10.1038/srep26124

58. Grande I, Berk M, Birmaher B, Vieta E. Bipolar disorder. Lancet (2016) 387:1561-72. doi: 10.1016/S0140-6736(15)00241-X

59. Vieta E, Berk M, Schulze TG, Carvalho AF, Suppes T, Calabrese JR, et al. Bipolar Disorders. Nat. Rev. Dis. Primers (2018) 4:18008. doi: 10.1038/ nrdp.2018.8

60. Berk M, Kapczinski F, Andreazza AC, Dean OM, Giorlando F, Maes M, et al. Pathways underlying neuroprogression in bipolar disorder: Focus on inflammation, oxidative stress and neurotrophic factors. Neurosci Biobehav Rev (2011) 35:804-17. doi: 10.1016/j.neubiorev.2010.10.001

Conflict of Interest: The authors declare that the research was conducted in the absence of any commercial or financial relationships that could be construed as a potential conflict of interest.

Copyright (c) 2020 Shan, Qiu, Pan, Teng, Li, Tang, Xiang, Wu, Tan, Chen, Guo, Wang and $W u$. This is an open-access article distributed under the terms of the Creative Commons Attribution License (CC BY). The use, distribution or reproduction in other forums is permitted, provided the original author(s) and the copyright owner(s) are credited and that the original publication in this journal is cited, in accordance with accepted academic practice. No use, distribution or reproduction is permitted which does not comply with these terms. 